\title{
Ruptura del abastecimiento de agua potable. Sistema Hampaturi-Pampahasi, La Paz, enero- febrero de 2008
}

Rupture de l'approvisionnement en eau potable. Système Hampaturi-Pampahasi, La Paz, janvier-février 2008

Breaking of the drinking water provision. The Hampaturi-Pampahasi system, La Paz, January-February 2008

\section{Sébastien Hardy}

\section{(2) OpenEdition}

Journals

Edición electrónica

URL: http://journals.openedition.org/bifea/2339

DOI: 10.4000/bifea.2339

ISSN: 2076-5827

\section{Editor}

Institut Français d'Études Andines

\section{Edición impresa}

Fecha de publicación: 1 diciembre 2009

Paginación: 545-560

ISSN: 0303-7495

Referencia electrónica

Sébastien Hardy, «Ruptura del abastecimiento de agua potable. Sistema Hampaturi-Pampahasi, La Paz, enero-febrero de 2008 », Bulletin de l'Institut français d'études andines [En línea], 38 (3) | 2009,

Publicado el 01 junio 2010, consultado el 18 noviembre 2020. URL : http://journals.openedition.org/ bifea/2339; DOI : https://doi.org/10.4000/bifea.2339

Les contenus du Bulletin de l'Institut français d'études andines sont mis à disposition selon les termes de la licence Creative Commons Attribution - Pas d'Utilisation Commerciale - Pas de Modification 4.0 International. 


\section{Ruptura del abastecimiento de agua potable. Sistema Hampaturi-Pampahasi, La Paz, enero-febrero de 2008}

Sébastien Hardy*

\section{Resumen}

El 25 de enero de 2008, un deslizamiento de terreno dañó una canalización de transporte de agua cruda entre la represa Hampaturi y la planta de tratamiento de Pampahasi. Durante diecinueve días, el tiempo de realizar trabajos de reparación de emergencia, más de un tercio de los habitantes de La Paz se quedaron sin abastecimiento de agua potable por la red de distribución. De igual estrago padecieron las empresas, los establecimientos de salud, las escuelas, etc. Este evento provocó unos disfuncionamientos muy importantes que llegaron a generar una crisis en parte de la ciudad.

Palabras clave: riesgo, vulnerabilidad, crisis, agua potable, La Paz

\section{Rupture de l'approvisionnement en eau potable. Système Hampaturi- Pampahasi, La Paz, janvier-février 2008}

\section{Résumé}

Le 25 janvier 2008, un glissement de terrain a endommagé une canalisation de transport d'eau crue entre le réservoir Hampaturi et l'usine de traitement de Pampahasi. Pendant dix-neuf jours, le temps de réaliser les réparations d'urgence, plus d'un tiers des habitants de La Paz ont été privés de l'accès au réseau de distribution en eau potable. Il en fut de même pour les entreprises, les établissements de santé, les écoles, etc. Cet évènement a ainsi créé dans une partie de la ville des dysfonctionnements très importants qui furent à l'origine d'une crise.

Mots clés : risque, vulnérabilité, crise, eau potable, La Paz

* Institut de Recherche pour le Développement (IRD), UR 029, programa Pacivur, Av. Hernando Siles 5290 esq. calle 7, CP 9214 Obrajes, La Paz, Bolivia. E-mail: sebastien.hardy@ird.fr 


\title{
Breaking of the drinking water provision. The Hampaturi-Pampahasi system, La Paz, January-February 2008
}

\begin{abstract}
On January 25, 2008, a landslide damaged a transport pipe of non-potable water between the Hampaturi reservoir and the treatment plant of Pampahasi. For nineteen days, the time required to complete the emergency repair work, more than one third of the inhabitants of La Paz were no longer supplied with drinking water by the distribution network, not counting companies, health care institutions, schools, etc. This event thus created important malfunctions in the the city that was the cause of a crisis.
\end{abstract}

Key words: risk, vulnerability, crisis, drinking water, La Paz

\section{DESCRIPCIÓN Y CONTEXTO DEL EVENTO}

El 25 de enero de 2008, un tramo de la canalización que transmite el agua cruda captada por Epsas S.A. (Empresa Pública Social de Agua y Saneamiento Sociedad Anónima), desde la zona de Hampaturi hacia la planta de potabilización de Pampahasi, fue dañado por un deslizamiento de tierra (fig. 1). Esto se explica a la vez por las condiciones geológicas de la zona de Hampaturi donde las tierras son blandas, y por las fuertes precipitaciones de días anteriores, normales durante la estación de lluvias (diciembre-marzo). Las precipitaciones tuvieron dos acciones paralelas: Ilenaron las represas de Epsas hasta su mayor límite de contención de agua. Por otro lado, las violentas ráfagas de viento durante la noche del 24 al 25 de enero hicieron desbordar el agua de la represa Janko Khota, situada aguas arriba de la cuenca vertiente del torrente Hampaturi, hacia las 3:00 de la mañana. Una ola de agua voluminosa y poderosa corrió cuesta abajo (entrevista con los mineros de La Solución, enero de 2009; entrevista con los técnicos de Epsas, enero de 2009) desencadenando a su paso el deslizamiento de tierra. Ya la estabilidad de las tierras había quedado comprometida por las precipitaciones de las anteriores semanas. El amontonamiento de materiales arrancados de las laderas creó una barrera en un lugar donde el lecho del torrente dibuja un codo. La violencia de su ruptura hacia las 4:00 de la mañana arrancó, aguas abajo, un pilar que permitía el paso aéreo del torrente a través de la canalización Hampaturi. Al hundirse, la canalización se partió en dos, dejando escapar el agua.

Mientras que los técnicos de Epsas detectaban la avería y cortaban la alimentación de agua de esa canalización, el derrame inundó los campos de la comunidad rural de Hampaturi, instalada en las cercanías. 
Ruptura de abastecimiento de agua potable, La Paz: enero-febrero 2008

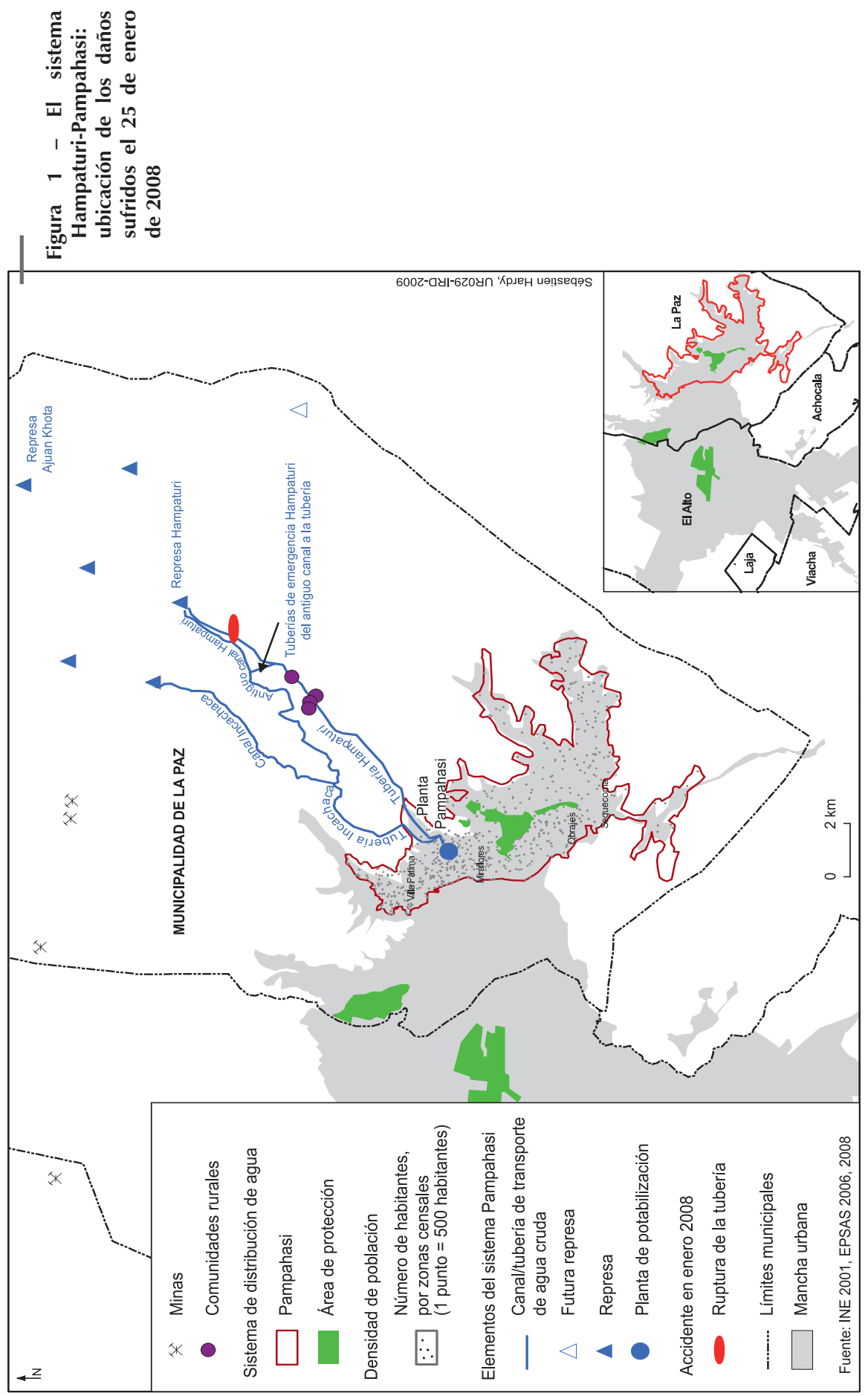




\section{CONSECUENCIAS DEL EVENTO}

A partir de las 10 de la mañana, este evento perturbó la producción de agua potable de la planta Pampahasi. La planta, que es alimentada en tiempo normal de agua cruda al mismo tiempo por la canalización Hampaturi $(400 \mathrm{l} / \mathrm{s})$ y por el canal Incachaca (300 l/s), ya no pudo inyectar las cantidades requeridas dentro de la red de distribución de agua potable. Solo aprovisionó a la red de agua potable con aproximadamente $300 \mathrm{l} / \mathrm{s}$ (canal Incachaca) en lugar de los $700 \mathrm{l} / \mathrm{s}$ normalmente distribuidos. Esta escasez de agua potable en la red de distribución generó graves disfuncionamientos de aprovisionamiento de agua en gran parte del sureste de la ciudad de La Paz abastecida por el sistema Hampaturi-Pampahasi.

Los disfuncionamientos duraron 19 días y el servicio se reanudó sólo a partir del 12 de febrero. Afectaron al menos a los 272000 habitantes que residen en el interior del sistema Hampaturi-Pampahasi, o sea el 34 \% de la población de La Paz conforme al censo de 2001, porcentaje que comprende a las poblaciones de las zonas residenciales más acomodadas de la aglomeración. Por lo general, su consumo de agua es elevado y saben imponer sus exigencias a las autoridades urbanas.

Además de los residentes, algunos de los elementos esenciales para el funcionamiento de la ciudad (D'Ercole \& Metzger, 2004) fueron afectados como 46 centros de salud de La Paz que suman 423 camas, los cuales se encontraron sin las cantidades necesarias de agua para el tratamiento de los pacientes (fig. 2).

La Cámara Departamental de Industria de La Paz señaló asimismo que las actividades económicas fueron perturbadas por la ruptura del aprovisionamiento de agua potable, un tipo de consecuencia que ya ha sido bien descrito en otros contextos (Clark \& Deininger, 2000: 75). Muchas empresas no recibieron las cantidades de agua que necesitaban para operar normalmente. Entre los barrios de Villa Fátima y Miraflores se encontraban unas 25 empresas cuyo consumo cotidiano de agua variaba entre 10000 y 1000000 litros por día. La falta de agua obligó a algunas de ellas a reducir sus actividades en un $50 \%$.

Otra consecuencia: la duración de los disfuncionamientos de aprovisionamiento obligó el Ministerio de Educación a postergar por una semana la entrada anual de las clases para todos los establecimientos educativos de La Paz, tanto privados como públicos (fig. 2). Esta postergación obligó muchos padres a pedir días de vacaciones excepcionales a sus empleadores para poder cuidar de sus hijos, lo que ocasionó gravámenes en el funcionamiento de algunas empresas.

Este panorama de algunas de las consecuencias de la ruptura de la canalización Hampaturi justifica que lo que podría considerarse a primera vista como un accidente únicamente de competencia de la empresa de producción y de distribución de agua potable de la aglomeración de La Paz, se convirtiera rápidamente en una crisis que afectó a gran parte de la población y de las actividades. No obstante, a pesar de marcados aspectos coyunturales, esta crisis se originó en los factores estructurales de vulnerabilidad de la aglomeración (Hardy, 2009a). 


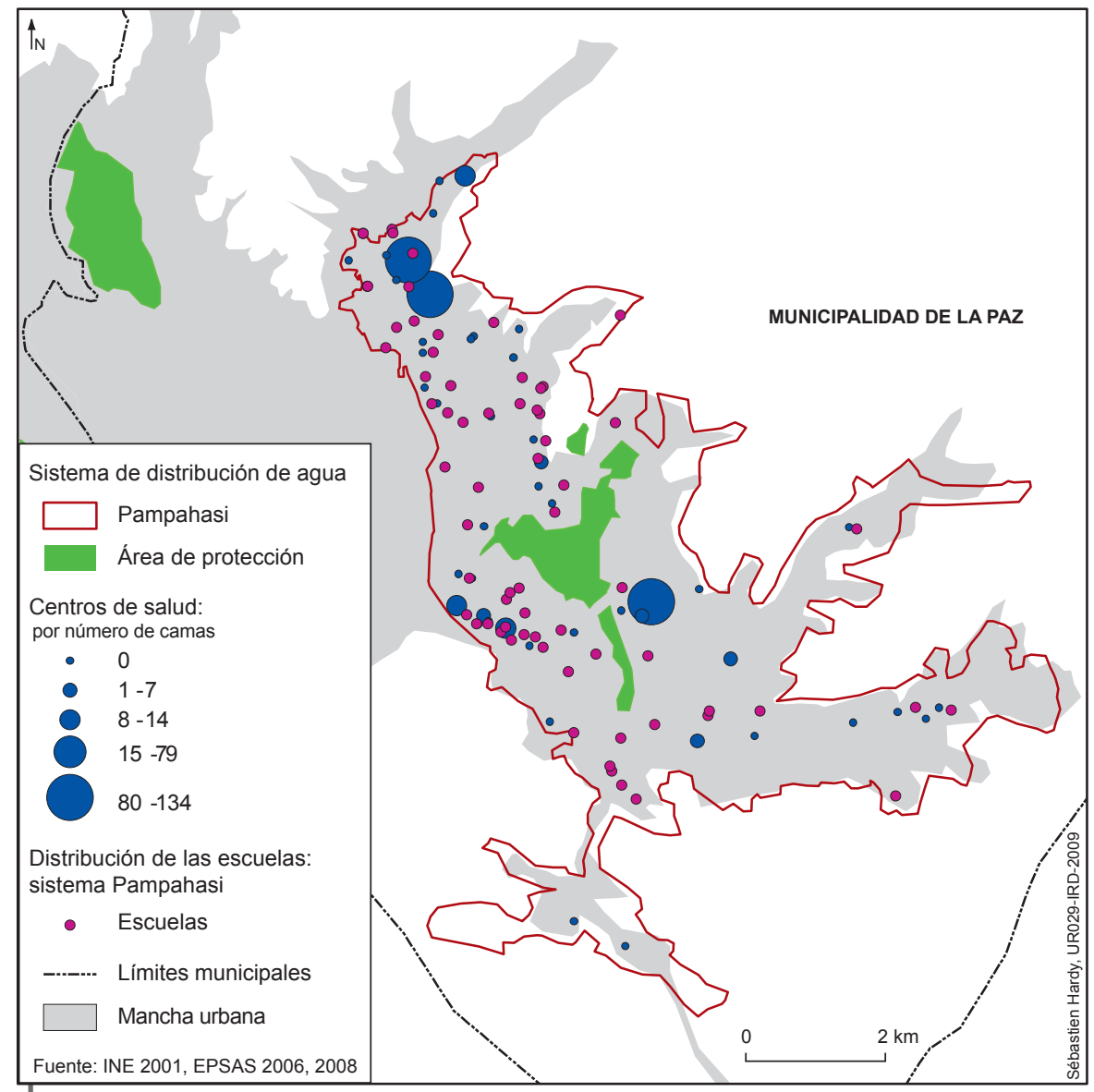

Figura 2 - Centros de salud y escuelas del sistema Hampaturi-Pampahasi afectados por la ruptura del aprovisionamiento de agua

\section{FACTORES DE VULNERABILIDAD}

Por un lado, parte de los elementos del subsistema Hampaturi-Pampahasi (represas de agua cruda, canales/canalización, estaciones reguladoras de energía, etc.) fue instalada en terrenos de condiciones geológicas poco propicias: alternancia de rocas permeables e impermeables que favorecen la saturación de agua de las capas superficiales y la formación de superficies de deslizamiento subyacentes; estructuras litológicas blandas. Por otro lado, los fuertes declives de la zona de Hampaturi aumentan los efectos erosivos de la escorrentía pluvial sobre tierras mal protegidas por una cobertura vegetal delgada. Finalmente, considerando que las precipitaciones se distribuyen en un periodo corto —el $70 \%$ de las precipitaciones anuales, es decir 572 mm en promedio, caen entre diciembre y marzo (Ramírez et al., 1995: 394) - y esencialmente en forma de lluvias torrenciales, se toma 
conciencia de las condiciones en las cuales se encuentran estos elementos y por qué son susceptibles de deterioro (Hardy, 2009b).

La instalación de los elementos del subsistema Hampaturi-Pampahasi en zonas de riesgo da origen a su vulnerabilidad. Esto tiene su explicación, entre otras, en la decisión, al comienzo del siglo XX, de producir y de distribuir el agua potable en grandes cantidades y a través de un gran sistema. En 2001, al menos el 83,6 \% de la población de la aglomeración de La Paz tiene así acceso a un agua potable de buena calidad y abundante, por medio de una red de distribución por canalización (Hardy, 2009a). Pero en un contexto de expansión espacio-demográfica de la aglomeración y del aumento del consumo de agua, hubo que aumentar los recursos de agua para garantizar el funcionamiento del gran sistema (Hardy, 2009a). La captación del agua cruda, que al principio se hacía en el torrente Choqueyapu en La Paz, tuvo que diversificarse. Ahora también se opera a partir de otras cuencas vertientes, todas ellas situadas al norte de la aglomeración y distantes de más de treinta kilómetros. Al extenderse, el gran sistema también ha multiplicado la exposición al daño de sus elementos de funcionamiento (Putnam, 2000), particularmente los de la producción, como en el caso del subsistema Hampaturi-Pampahasi.

Al analizar el sistema desde la perspectiva de la red de distribución de agua aparecen también factores de vulnerabilidad estructural. En primer lugar hay que precisar que la red de distribución de agua potable del subsistema Hampaturi-Pampahasi tiene un modo de funcionamiento centrífugo: el agua es distribuida desde la planta de potabilización de Pampahasi (a través de represas de almacenamiento) hasta los extremos de la red. Desde allí el agua potable es distribuida de manera lineal a partir de dos canalizaciones principales: una de dirección suroeste, de un diámetro de 600 mm; la otra de dirección sureste, de un diámetro de 700 mm. Las redes secundarias de distribución se ramifican enseguida a partir de dos canalizaciones principales. El agua por lo tanto está disponible para los consumidores de la parte aguas arriba de la red, y progresa enseguida hacia aguas abajo y los extremos de la red. En caso de mínimas entradas de agua potable en la red, las partes aguas abajo corren el riesgo de no recibir las cantidades suficientes para cubrir sus demandas. Por ultimo, ya que la red de distribución funciona por gravedad, la disminución de la cantidad de agua reduce también la presión y por ende la capacidad de distribuir el agua en las partes elevadas de la red.

En el caso de escasez de agua, como en el evento de enero-febrero de 2008, la falta de alternativa que ofrece este modo de funcionamiento del subsistema Hampaturi-Pampahasi vuelve vulnerables ciertas partes de la red de distribución, y por lo tanto ciertos elementos del funcionamiento urbano que requieren de agua. Vislumbramos aquí las transmisiones transversales de vulnerabilidad y los espacios de riesgo.

Por otro lado, la vulnerabilidad de aprovisionamiento de agua potable del subsistema Hampaturi-Pampahasi puede transmitirse a elementos cuyo funcionamiento depende mucho del agua potable, por ejemplo los centros de salud. Efectivamente, a causa de los disfuncionamientos del subsistema, los centros de salud abastecidos por éste pueden ser afectados en su buen funcionamiento. 
Comprenderlo permite aprehender las transmisiones de vulnerabilidad, de un elemento del funcionamiento urbano (sistema de abastecimiento de agua potable) a otro (establecimiento de salud), y por ende los territorios de riesgo (lugares y espacios dañados)1.

Por otro lado, la empresa responsable de la producción y de la distribución del agua potable no tiene verdaderamente una gestión proactiva que le permita anticipar los problemas y administrarlos mejor. Aunque es posible identificar el vínculo entre esta vulnerabilidad y la gestión de la empresa, no obstante está relacionada ante todo a la vulnerabilidad institucional que la caracteriza. La empresa fue nacionalizada en enero de 2007 luego del retiro, negociado por el gobierno de Evo Morales, del accionista francés Suez. La empresa, que ahora tiene un estatuto de sociedad anónima, sigue en proceso de restructuración (Poupeau, 2008). No tiene los medios humanos ni financieros que le permitan garantizar la seguridad del abastecimiento de agua potable (Hardy, 2009a).

La empresa ha heredado anomalías de orden jurídico con las cuales tiene que lidiar (Pozo et al., 2006). Efectivamente, los elementos de producción y de distribución de agua están ubicados en terrenos en los cuales no siempre es propietaria. La reforma agraria de 1953 modificó la situación para la empresa concesionaria del servicio de agua: los terrenos inicialmente de propiedad pública y de uso colectivo pasaron al estatus privado cuando habían sido instaladas infraestructuras públicas. El uso hizo que la empresa, actualmente privada, tuviera normalmente acceso a las instalaciones de las que es concesionaria (derecho de servidumbre). No obstante, no se perciben aún todas las consecuencias de esta situación jurídica, lo que hace que ciertos elementos del sistema de aprovisionamiento de agua potable sean vulnerables. Por ejemplo, al ser el control de la urbanización muy ineficiente en la aglomeración, no ha sido posible a menudo definir franjas de seguridad alrededor de las instalaciones sensibles para el funcionamiento urbano. Se atribuyeron títulos de propiedad cuando los terrenos en cuestión contaban con las instalaciones de la empresa de agua. Hoy, algunos de los pozos que explota esta en El Alto están ubicados dentro de propiedades urbanas (Hardy, 2009a). Aunque el uso hace que la empresa acceda a las instalaciones en virtud del derecho de servidumbre, el buen funcionamiento de ciertos elementos del sistema está un poco comprometido, simplemente por mínima facilidad de control. Sobre todo las poblaciones han tomado progresivamente conciencia de este factor de vulnerabilidad de la empresa. No dudan en jugar con ello principalmente para obtener de ésta ventajas o compensaciones por el uso y el derecho de acceso. Presa y sin apoyo jurídico en el cual apoyarse, sobre todo cuando se trata de una situación de urgencia, con frecuencia la empresa no tiene otra salida que negociar con la población.

En enero-febrero de 2008, todos y cada uno de estos factores estructurales de vulnerabilidad han jugado un rol y frecuentemente han interactuado entre ellos, facilitando finalmente la situación de crisis por transmisión de los disfuncionamientos a otros elementos del funcionamiento urbano que se vieron afectados: población, sistema de salud, sistema educativo y sistema económico. 


\section{GESTIÓN DE CRISIS}

Un análisis retrospectivo del evento permite resaltar los factores específicos que, junto con los factores estructurales de vulnerabilidad, finalmente han conducido a la crisis. Entre ellos se encuentran aquellos de competencia exclusiva de la empresa de producción y de distribución de agua potable, y los que, aunque externos al funcionamiento de la empresa, han formado ampliamente parte de la situación de crisis.

\section{1. Los factores coyunturales de crisis que dependen de Epsas}

En primer lugar, los disfuncionamientos de aprovisionamiento de agua tienen su explicación en los daños de un elemento del sistema de producción de agua: la canalización de transporte del agua cruda captada en la cuenca vertiente de Hampaturi. Asimismo, la solución parece ser de orden técnico, del campo de competencia de la empresa de agua. Pero la reparación de esta canalización ha resultado compleja. Después de haber anunciado 2 meses de trabajos (el 26 de enero), Epsas tuvo que reevaluarlos en 5 meses (el 1 de febrero). Mientras tanto, Epsas pensó ser capaz de restablecer muy rápidamente un servicio de distribución de agua potable por red utilizando alternativas para la canalización dañada. El 26 de febrero, la empresa anunciaba el restablecimiento del servicio en menos de 48 horas.

En un primer tiempo, Epsas preveía hacer pasar agua de la represa Hampaturi a la planta de Pampahasi, no utilizando más la canalización Hampaturi que cumplía esta función (1945-1994) antes de la construcción de la nueva (fig. 3). De esta manera, la planta habría podido recibir de nuevo sus $700 \mathrm{l} / \mathrm{s}$ de agua cruda (300 l/s por Incachaca y $400 \mathrm{l} / \mathrm{s}$ por el antiguo canal Hampaturi), necesarios para la alimentación de la red de distribución. Sin embargo, la rehabilitación del conjunto del canal Hampaturi ha resultado imposible. Dado que este canal fue abandonado hace 15 años y no pudo ser suficientemente mantenido para soportar una completa puesta en servicio (tramos arrancados por deslizamientos de tierra, etc.), Epsas tuvo que reorientarse hacia otra solución técnica en dos tiempos:

1) transferir el flujo de agua cruda de la represa Hampaturi hacia el antiguo canal Hampaturi (400 l/s como máximo), el tiempo de eludir la ruptura;

2) seguidamente transportar el flujo de agua hacia la canalización Hampaturi a través de una canalización de urgencia.

Los trabajos para instalar esta canalización de urgencia fueron decididos el 30 de enero, pero no pudieron realmente iniciarse sino el 4 de febrero. En este caso también la solución técnica resultó un poco más difícil de ejecutar de lo previsto, debido a condiciones geológicas y también al contexto sociopolítico boliviano.

La transferencia del agua desde el antiguo canal hacia la canalización Hampaturi, aguas abajo del punto de ruptura, ha movilizado todas las energías. Epsas no ha cesado de aumentar el número de obreros asignados a los trabajos de 


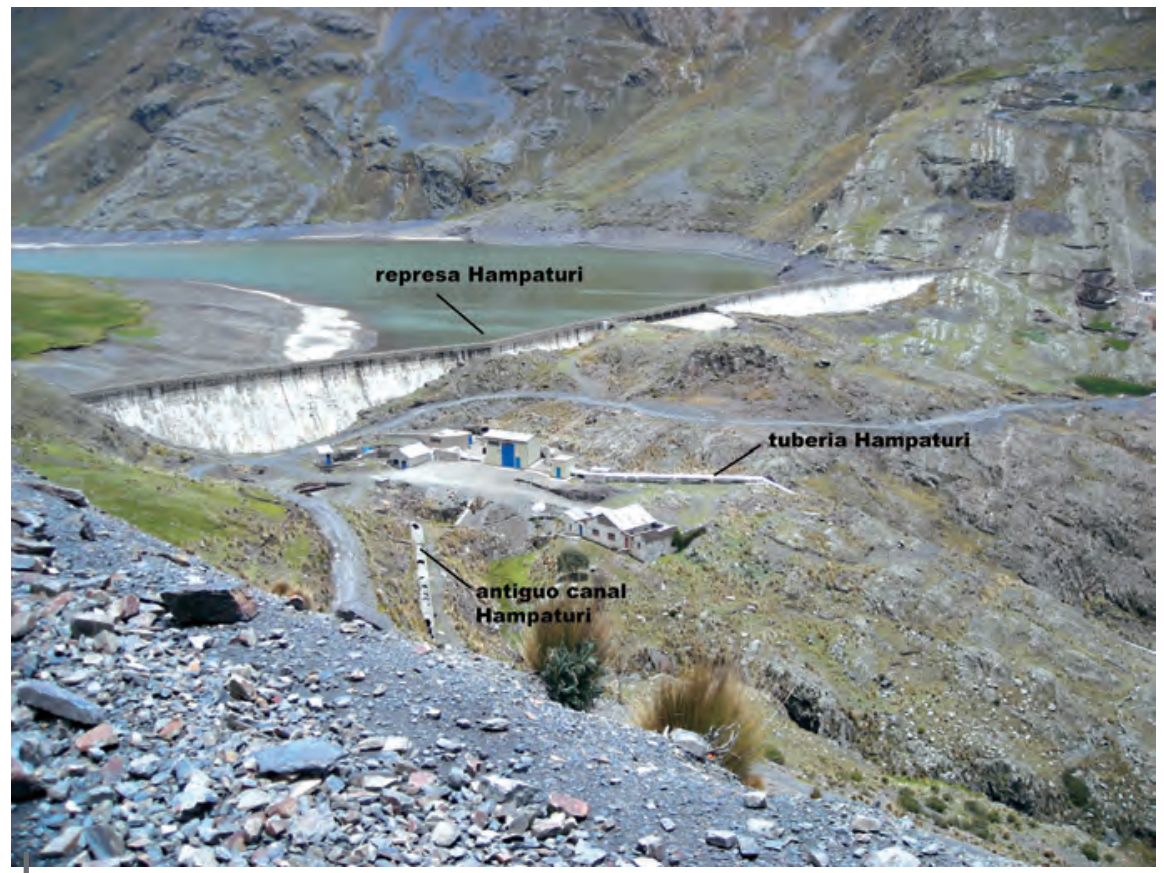

Figura 3 - Una parte de los elementos del sistema Hampaturi-Pampahasi

Foto S. Hardy, enero de 2009

la canalización de urgencia. Recibió el apoyo, desde el 6 de febrero, de las autoridades políticas. El número de trabajadores pasó de 50 a 250: empleados de Epsas (90) y militares (130 conscriptos del Regimiento de Infantería Colorados de Bolivia, 30 soldados del Batallón de la Policía militar de la Fuerza Naval Boliviana). La alternativa pareció operativa el martes 12 de febrero, la cual fue inaugurada por el Presidente de la República. Pero el aprovisionamiento de la planta de Pampahasi fue de nuevo interrumpido los días 15, 16 y 17 de febrero. Esto se debe a la rapidez con la que se efectuaron los trabajos lo cual afectó su calidad: aparecieron fisuras debidas a la presión en la canalización de urgencia que tuvo que ser parcialmente reemplazada. De manera general, a pesar del buen funcionamiento de la alternativa, la vulnerabilidad del aprovisionamiento de agua del subsistema Hampaturi-Pampahasi se mantuvo alta hasta el 18 de julio, fecha en la cual la canalización dañada fue puesta nuevamente en servicio (GMLP, 2008). Hasta aquel entonces, el subsistema no había podido recuperar sino un caudal de aproximadamente $600 \mathrm{l} / \mathrm{s}$. Esta dotación solo representaba el 85 \% del caudal necesario para su funcionamiento normal.

Ante las dificultades técnicas para transferir el agua cruda del antiguo canal hacia la canalización, y ante el aumento resultante de las dificultades para abastecer de agua potable a la parte sureste de La Paz, Epsas tuvo rápidamente que admitir las cosas tales como eran: no podía mantener el plan de distribución de agua 
establecido el 26 de enero, y que debía funcionar durante 48 horas: la distribución de agua por la red, mediante rotaciones de 12 horas; y el resto del tiempo, una distribución de urgencia de agua potable utilizando camiones cisternas abastecidos por los otros subsistemas (Achachicala y El Alto). Con respecto a la distribución, la empresa se enfrentó a dos grandes problemas, por falta de anticipación de una posible crisis y sus consecuencias.

Por una parte, el modo de funcionamiento de la red de distribución hacía que el agua estuviera disponible primero para los consumidores de la parte aguas arriba de la red. Siendo los primeros en ser servidos, éstos utilizaron el caudal máximo de los $300 \mathrm{l} / \mathrm{s}$ disponibles en la red durante todo el período de la crisis para cubrir sus necesidades. Las partes aguas abajo se encontraron a menudo sin ninguna dotación, principalmente los extremos de la red. Epsas hizo lo posible por repartir mejor la dotación disponible imponiendo rotaciones horarias. Pero la operación era técnicamente difícil: demasiadas compuertas que abrir y cerrar en poco tiempo para individualizar mejor la distribución en un espacio tan vasto.

Por otro lado, los habitantes no fueron muy solidarios como para aceptar una disminución de su consumo en beneficio de una dotación mínima para un mayor número de personas. Epsas les había pedido reducir su consumo de agua. Pues bien, la parte de la ciudad abastecida por el sistema Pampahasi concentra zonas residenciales más acomodadas donde los consumos de agua son voluminosos. Estas poblaciones tuvieron dificultades para disminuir sus necesidades de agua, por ejemplo diferenciando los usos de agua: potable (para beber y cocinar) y no potable (baños y limpieza). Por lo demás, desde los primeros días de la ruptura, los precios de venta del agua potable en botella aumentaron considerablemente frente a la demanda. La ruptura sucedió en un momento del año en que las temperaturas son más elevadas, lo que indujo mayor consumo de agua potable. Además, la crisis tuvo lugar durante el periodo de precarnaval. Tradicionalmente, los bolivianos y en particular los jóvenes y niños se divierten tirándose agua. A pesar de las dificultades para aprovisionarse de agua los juegos de agua se llevaron a cabo durante todo el periodo de crisis, incluso en la parte afectada de la ciudad.

Por otra parte, la empresa no tenía ningún conocimiento de los lugares más eficaces para ubicar sus camiones cisternas y abarcar a un mayor número de habitantes, en función de la distribución de las densidades por ejemplo. La empresa había elegido puntos de distribución de agua por camiones cisternas, anunciados a través de los medios de comunicación, pero sin tomar en consideración las necesidades de las poblaciones en función de los diferentes espacios donde se daría el servicio. Rápidamente estas rotaciones mostraron ser poco eficaces para distribuir el agua a los habitantes afectados porque la ubicación de los lugares elegidos para poner a disposición las cisternas fue imprecisa y además los horarios previstos no se respetaron; finalmente, la empresa no disponía de volúmenes en cisternas necesarios para aprovisionar a 272000 habitantes en un lapso de tiempo que no cesó de aumentar. De esta manera, Epsas tuvo que pedir ayuda externa: camiones cisternas de los bomberos, del Gobierno Municipal de La Paz (GMLP) y de la Sabenpe (empresa encargada de la limpieza en La Paz), así como los camiones cisternas pertenecientes a particulares. En total, 16 camiones cisternas 
con capacidades comprendidas entre 8000 y 20000 litros distribuyeron agua durante los 19 días de la crisis. Pero a causa de la heterogeneidad de sus orígenes, Epsas nunca supo planificar con anticipación el número exacto de camiones cisternas disponibles cada día para efectuar las distribuciones. Las cantidades de agua distribuidas no eran suficientes para cubrir las necesidades.

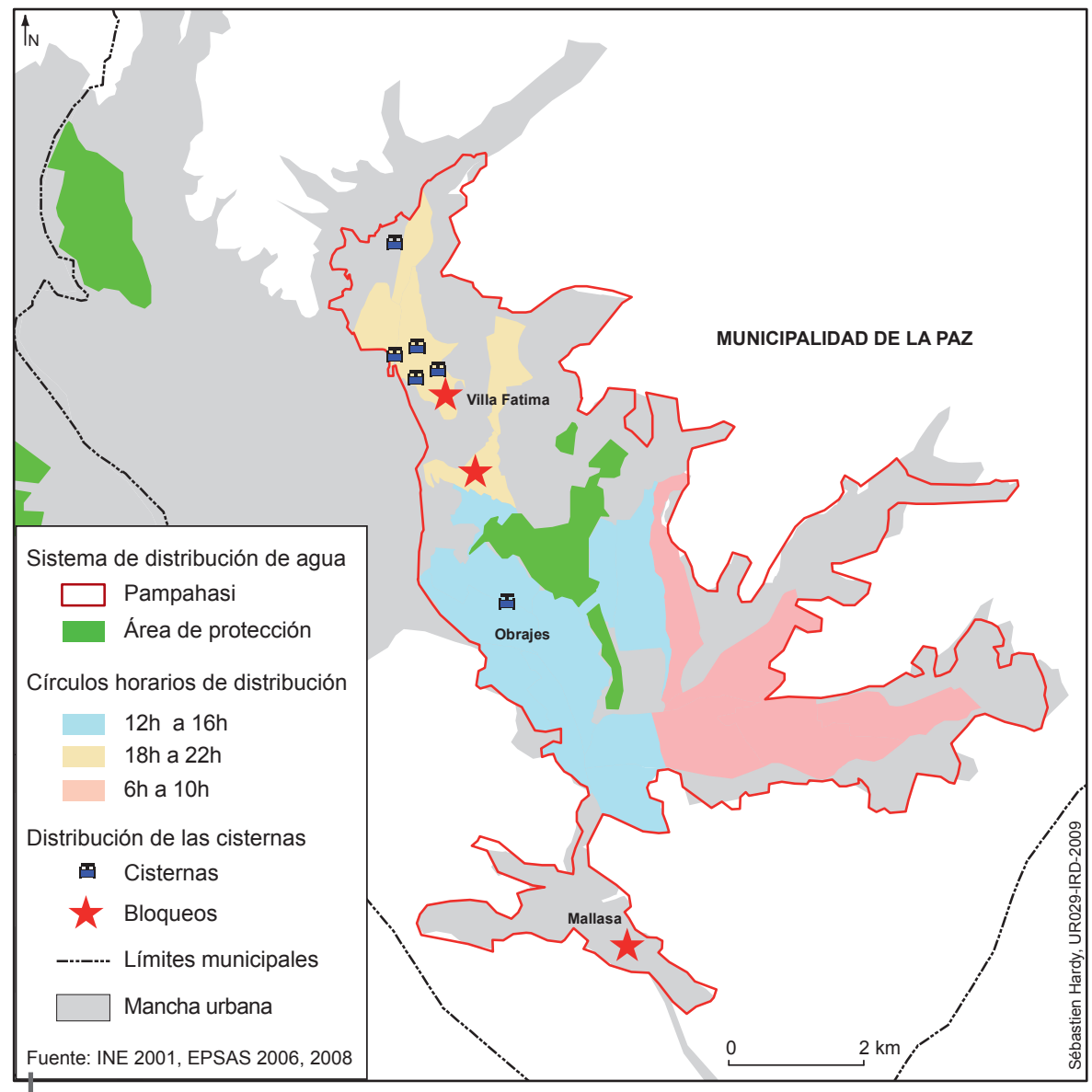

Figura 4 - La gestión de crisis: rotaciones de la distribución de agua por red, puntos de aprovisionamiento por camiones cisternas y puntos de conflictos

Frente a la insuficiencia de las cantidades de agua distribuidas y al malestar cotidiano que la ruptura de aprovisionamiento generó, y ante las dificultades de la empresa para comunicar correctamente su plan de contingencia, la población perdió paciencia e interpeló a las autoridades desde el 27 de enero, bloqueando ciertas vías (fig. 4). A veces su descontento se tradujo en la toma por asalto de los camiones cisternas destinados a dar servicio a otros barrios. Se observó también la degradación de las instalaciones de distribución de Epsas para procurarse agua, como en Villa Fátima el 28 de enero. 
La empresa se vio obligada a readaptar varias veces su plan de distribución de urgencia de agua potable. Desde el 28 de enero se dispuso de agua por red solo 8 horas por día, y se distribuyó el agua por cisterna el resto del tiempo. El 2 de febrero, para paliar la baja presión en la red de distribución, Epsas se vio obligada a reducir las horas de distribución de agua a 4 horas por día (fig. 4), manteniendo la dotación de agua potable por camiones cisternas. Paralelamente, la empresa procedió a una transferencia de agua desde el subsistema de distribución de agua potable El Alto para aprovisionar puntualmente ciertos barrios como Obrajes y Següencoma. A pesar de la combinación de todas estas medidas, la dotación de agua se mantuvo baja hasta la entrada en servicio de la canalización de urgencia el 18 de febrero (que sin embargo solo permitió restablecer el 85 \% del caudal necesario para el funcionamiento normal).

\section{2. Los factores coyunturales de crisis que escapan a las competencias de Epsas}

Leyendo lo anterior, se observa que la empresa de agua ha reaccionado a lo que consideró como un simple accidente sobre un elemento del sistema de producción. De cualquier manera solo tenía competencia para intervenir en este aspecto. Los habitantes de La Paz han criticado mucho esta gestión de la crisis por parte de Epsas, pero han olvidado rápidamente que muchos aspectos de la crisis, por ser exteriores a la empresa, estaban fuera de su control. Un análisis retrospectivo del evento permite poner en evidencia este hecho y también comprender de qué manera el contexto sociopolítico boliviano ha pesado enormemente en esta crisis.

A pesar de las condiciones de difícil acceso a la zona del accidente, a causa de los caminos de tierra impracticables por las fuertes lluvias, el gerente de Epsas lo fue a visitar el 25 de enero para evaluar la magnitud de los daños y estudiar las posibles soluciones a fin de restablecer el abastecimiento de agua. Entonces fue interpelado por campesinos de las comunidades rurales de Hampaturi que protestaban contra los daños sufridos por sus cultivos y sus viviendas, a raíz de la inundación que había resultado de la ruptura de la canalización. Las comunidades rurales reclamaban a la empresa compensaciones por los daños provocados a cambio del acceso a la zona para poder efectuar las reparaciones necesarias. El evento sucedió el fin de semana y las autoridades responsables no midieron su importancia de inmediato — principalmente en sus componentes sociales - ni la urgencia de una respuesta inmediata. En descargo de la empresa, la imprecisión de su situación jurídica no le daba la posibilidad de iniciar negociaciones con los habitantes de las comunidades rurales.

Poco a poco, Epsas y las autoridades políticas tomaron consciencia de la magnitud de la crisis que se presentaba, y que no era únicamente de orden técnico. Entonces emprendieron negociaciones con los habitantes de las comunidades rurales de Hampaturi a fin de permitir a los obreros de acceder a la canalización deteriorada. El Secretario general del GMLP intervino el 28 de enero para lanzar estas 
negociaciones. Con este fin reunió a los representantes de la Central agraria La Cumbre, a la alcaldesa adjunta de Hampaturi, a la consejera municipal representante de Hampaturi y al gerente de Epsas. Los habitantes de las comunidades campesinas reclamaban el pago de derechos por el paso de la canalización de Epsas a través de sus tierras. La ausencia de estatuto jurídico de Epsas le impidió negociar sola y por ende con rapidez. Por lo cual las autoridades políticas municipales y nacionales intervinieron de manera más visible en la gestión de la crisis.

Fue así que el presidente de la República, acompañado por el alcalde de La Paz, se presentó el 3 de febrero en la zona de Hampaturi para explicar a los habitantes la importancia de los trabajos que debían efectuarse con urgencia. Obtuvo entonces la firma de un preacuerdo entre las autoridades públicas (Ministerio del Agua, Ministerio de Desarrollo Rural y Ministerio del Interior) y los representantes de los habitantes, el cual contemplaba entre otros las realización de diques defensivos en los torrentes Hampaturi y Palcoma, con el fin de proteger las viviendas y las zonas de cultivo de las frecuentes crecidas. La firma de este preacuerdo marcó el comienzo de las reparaciones.

La implicación de las autoridades se tradujo también en el plano económico. Efectivamente, durante una reunión con el alcalde de La Paz, los responsables de Epsas, ingenieros militares y el ministro del Agua, se informó al presidente de la República que el costo de las reparaciones de la canalización de Hampaturi era de por lo menos 450000 \$US. Epsas no estaba en posibilidad de financiar tales trabajos. Frente a la magnitud de la crisis, el presidente de la República decidió solicitar con urgencia un crédito a la Corporación Andina de Fomento (CAF). Pero Epsas, habiéndose constituido como una sociedad anónima, no podía beneficiarse de una transferencia de presupuesto del Estado. Por lo tanto fue el GMLP el que recibió el préstamo y el que administró los trabajos de reparación. El alcalde de La Paz, apoyándose en el Decreto Supremo de urgencia nacional firmado por el presidente de la República para hacer frente a las diferentes catástrofes que sufría el país en aquel momento, decidió recurrir el 4 de febrero a una adjudicación por excepción. La reparación de la canalización dañada fue entonces atribuida a la empresa TAURO S.A.

Durante todo el periodo de crisis, los consumidores tuvieron que adaptarse a la situación encontrando alternativas. Esta capacidad de adaptación es uno de los factores que permiten medir la vulnerabilidad. Por ejemplo, el 25 de febrero, el centro de salud Prosalud de Villa Fátima se vio en la obligación de pedir agua a los habitantes instalados más abajo con el fin de no suspender su funcionamiento. El caso de ciertos hospitales, encargados principalmente de realizar las hemodiálisis para el conjunto de la ciudad de La Paz, es más inquietante. Únicamente para este tratamiento un hospital como Arco Iris necesita cada día 45000 litros de agua. Pues bien, entre el 25 y el 27 de enero, solo habría recibido 36000 litros de agua gracias a los camiones cisternas especialmente enviados por Epsas. Este ejemplo permite subrayar dos aspectos de la vulnerabilidad: por una parte la vulnerabilidad organizativa de la empresa en situación de urgencia para garantizar el aprovisionamiento de agua de elementos esenciales al funcionamiento urbano como los hospitales; por otra parte, la vulnerabilidad que se transmite por la dependencia de agua potable 
de la red, de ciertos elementos como los establecimientos de salud. Las mínimas cantidades de agua potable que recibieron fragilizaron la calidad de los tratamientos propuestos (cf. supra).

El análisis de la vulnerabilidad por capacidad de adaptación puede realizarse sobre otros elementos esenciales como las empresas, a veces muy dependientes del aprovisionamiento de agua para garantizar su funcionamiento. Algunos habían anticipado estos posibles disfuncionamientos asegurándose una fuente alternativa de abastecimiento de agua mediante de un pozo privado. Otros supieron adaptarse durante la crisis: la empresa textil Universaltex, situada en el barrio Petrolero, consume 1000000 litros de agua por día para fabricar 6 toneladas de tela; durante el periodo de ruptura del aprovisionamiento de agua solo producía 4 toneladas. Para funcionar, la empresa había suprimido uno de los tres ciclos cotidianos de trabajo y se había aprovisionado parcialmente de agua comprándola a camiones cisternas privados que a veces venían de la ciudad de Oruro, a 240 kilómetros de distancia de La Paz.

Algunas poblaciones mostraron ser capaces de encontrar alternativas. La prensa reveló algunas: proveerse de agua con conocidos que no vivían en la zona del subsistema Pampahasi — probando la importancia de un mantenimiento activo de redes sociales_- utilizar los recursos de agua de su empresa, mostrando eventualmente al empleador las ventajas que podía sacar (como el no ausentismo de sus empleados); utilizar las fuentes resurgentes del acuífero del altiplano, distribuidas por todos lados en las laderas gratuitamente (La Razón, 2008). En cambio, otros habitantes se mostraron menos aptos para hacer frente a la situación de crisis. En la parte más rica del sector afectado, las poblaciones no tenían los recipientes adecuados para almacenar el agua distribuida por los camiones cisternas, como en barrios más populares mejor preparados para los cortes de agua. Esto obligó los camiones cisternas de Epsas a que regresaran varias veces por día a distribuirles agua, en detrimento de otros barrios.

\section{INCIDENCIAS DEL EVENTO}

El análisis de la crisis no se detiene en el periodo mismo de los eventos. Este obliga a interesarse también en sus incidencias, en particular en términos de prevención de las vulnerabilidades que la crisis puso de manifiesto.

A partir del momento en el cual las soluciones alternativas de aprovisionamiento funcionaron de manera suficientemente regular para alimentar de agua potable a la parte sureste de la ciudad, los responsables de la empresa y las autoridades municipales, más tranquilos, se ocuparon de examinar la vulnerabilidad del sistema Pampahasi. Detectaron que al menos 300 metros lineales de canalización se situaban sobre tierras inestables, y que fisuras eran visibles en una estación reguladora de presión. Por esta razón el alcalde de La Paz hizo llegar un informe técnico al presidente de la República y a Epsas, para que se llevaran a cabo disposiciones a fin de efectuar las reparaciones preventivas necesarias. 
La crisis también fue explotada para denunciar la ausencia de estatuto de la empresa de producción y de distribución del agua, más de un año después de su nacionalización. Por ejemplo, el alcalde de La Paz, apoyado por los presidentes de las Federaciones de juntas de vecinos (Fejuve) de La Paz y de El Alto, ha denunciado públicamente la incapacidad de Epsas para administrar por delegación, técnicamente y económicamente, un servicio público destinado a una aglomeración importante como La Paz. Denunció el estancamiento de los trabajos de la Comisión interinstitucional, que había sido creada en enero de 2007 para definir el estatuto de Epsas, y su modo de financiamiento. Ante la magnitud de los problemas revelados por esta crisis, el alcalde de La Paz, apoyado esta vez por jefes de empresas paceñas, mencionó la necesidad de dotar Epsas de un estatus de empresa mixta para garantizar las inversiones necesarias para su buen funcionamiento, y reducir su vulnerabilidad institucional y técnica. Mientras la legitimidad de la ciudad como sede del gobierno es regularmente cuestionada por las ciudades de Santa Cruz y de Sucre (Hardy, 2009c), para las autoridades municipales, el buen funcionamiento de la ciudad a través de un servicio tan importante como el abastecimiento de agua potable constituye por lo tanto un desafío. Sin embargo, las autoridades gubernamentales, conscientes del desafío, rehusaron la constitución de una empresa mixta. A pesar de lo que reveló la crisis, uno se da cuenta que la política de prevención de los riesgos ligados al sistema de aprovisionamiento de agua potable escapa parcialmente a Epsas, y que los factores de vulnerabilidad siguen sin solución inmediata, teniendo en cuenta el contexto político nacional.

En definitiva, la crisis ha debilitado económicamente a Epsas. La autoridad reguladora (Sisab) la ha obligado a no facturar el agua potable distribuida por camiones cisternas durante los 19 días que duró la crisis, a pesar del costo que esta operación de urgencia representó para la empresa.

Por lo tanto la empresa y los habitantes pueden sacar muchas enseñanzas de esta crisis para reducir las vulnerabilidades del sistema de aprovisionamiento de agua potable y sus transmisiones a otros elementos. Buen ejemplo constituye lo que se hizo en San Francisco donde el terremoto de 1906 aún continúa alimentando la reflexión de los responsables del aprovisionamiento de agua potable (Putnam, 2000; Bruins, 2000). Una de las enseñanzas se refiere a los espacios. El espacio del daño ha generado espacios de riesgos donde la crisis ha debido ser manejada. Conviene recalcar que la identificación de estos espacios permite anticipar las vulnerabilidades. El evento ha mostrado además que los espacios de la crisis pueden ser múltiples: lugar del daño que también es el lugar del conflicto con las comunidades rurales de Hampaturi; lugares de los problemas de distribución de agua potable en la ciudad de La Paz. El análisis de estos espacios de la crisis y de su gestión permite poner de relieve los elementos esenciales que hay que proteger, sus vulnerabilidades, para prevenir futuros disfuncionamientos posibles. Finalmente, esta crisis ha permitido iniciar una reflexión en términos de alternativas: fue el caso para Epsas pero también para la población: ambas tuvieron que pensar en alternativas de abastecimiento de agua potable (Clark \& Deininger, 2000; Putnam, 2000; D’Ercole \& Metzger, 2004). La búsqueda de estas alternativas ha vuelto un 
poco menos vulnerables a las poblaciones frente a un futuro disfuncionamiento del aprovisionamiento de agua potable, preparándolas. Esta conclusión puede extenderse al sector económico y al sector de la salud.

\section{Referencias citadas}

BRUINS, H., 2000 - Proactive Contingency Planning vis-à-vis Declining Water Security in the 21st Century. Journal of contingencies and crisis management, vol. $\mathbf{8}, \mathbf{n} .^{\circ} \mathbf{2}$ : 63-72.

CLARK, R. \& DEININGER, A., 2000 - Protecting the Nation's Critical Infrastructure: The Vulnerability of U.S. Water Supply Systems. Journal of contingencies and crisis management, vol. 8, . $^{\circ}$ 2: 73-80.

D'ERCOLE, R. \& METZGER, P., 2004 - Vulnerabilidad del Distrito Metropolitano de Quito, 496 pp.; Quito, Ecuador: MDMQ-IRD. Colección Quito Metropolitano.

GMLP, 2008 - Notas del día, URL < http://lapaz/ES/Paginas/default.htm>, del 25 de enero 2008 al 18 de julio 2008.

HARDY, S., 2009a - La vulnérabilité de l'approvisionnement en eau dans I'agglomération pacénienne. Le cas du sous-système El Alto. Cybergéo : European Journal of Geography, [En línea], Dossiers, Vulnérabilités urbaines au sud, document 457, mis en ligne le 20 mai 2009. URL : http://www.cybergeo.eu/index22270.html

HARDY, S., 2009b - Explorer la construction de la résilience. Expériences de recherche à La Paz. In: Vulnérabilités sociétales, risques et environnement (A. Pelier \& S. Becerra, eds.): 469-482; París: Editions L'Harmattan.

HARDY, S., 2009c - Enjeux et fractures de la Bolivie en 2009. EchoGéo, Sur le vif 2009, [En ligne], URL : http://echogeo.revues.org/index10965.html.

La Razón, 2008 - URL < http//www.larazon.com.bo>, del 25 de enero 2008 al 25 de febrero 2008.

POUPEAU, F., 2008 - Les ambivalences de la participation communautaire. Le cas du service de distribution des eaux à El Alto, Bolivie. Autrepart, n. ${ }^{\circ}$ 47: 245-253.

POZO, E. et al., 2006 - Informe final a la Auditoría Regulatoria con carácter TécnicoAmbiental, Económico-Financiera, Legal y Comercial sobre el cumplimiento del Contrato de Concesión de Aguas del Illimani S.A. Resumen ejecutivo, 55 pp.; La Paz: Pozo \& Asociados SRL.

PUTNAM, D., 2000 - Earthquakes and Water Security: Contingency Planning in California. Journal of contingencies and crisis management, vol. 8, n. ${ }^{\circ}$ 2: 103-108.

RAMÍREZ, E., MENDOZA, J., SALAS, E. \& RIBSTEIN, P., 1995 - Régimen espacial y temporal de las precipitaciones en la cuenca de La Paz. Bulletin de l'Institut Français d'Études Andines, 24 (3): 391-401. 